\title{
Implantation of permanent pleural catheter for palliation of malignant pleural effusion
}

\author{
Michelle Meier' \\ Marie Riis Mortensen' \\ Lene Unmack Larsen² \\ 'Department of Surgical \\ Gastroenterology, ${ }^{2}$ Department of \\ Radiology, Aarhus University Hospital, \\ Aarhus, Denmark
}

This article was published in the following Dove Press journal:

Cancer Management and Research

8 November 2016

Number of times this article has been viewed

Purpose: Malignant pleural effusion (MPE) is a disabling condition caused in most instances by far-advanced cancer. The treatment is palliative and should ideally be minimally invasive. The aim of this retrospective study was to evaluate the effectiveness of implantation of a permanent PleurX catheter in the treatment of recurrent MPE in patients with terminal cancer disease.

Patients and methods: Eighteen consecutive patients with terminal cancer and MPE, who had a permanent PleurX catheter implanted in the period from February 2014 to August 2015, were retrospectively evaluated. Medical records were reviewed for data on procedures and patients. Results: Twenty-two PleurX catheters were implanted in the pleural cavity of 18 patients. The technical success rate was $100 \%$. All patients had relief of symptoms. Catheter patency was $91 \%$. Six patients experienced minor adverse events (AEs) and two patients experienced moderate-tosevere AEs. The median survival time for the 14 patients who died during follow-up was 45 days. Conclusion: Implantation of a permanent PleurX catheter is a safe procedure with a high success rate and only few AEs. The procedure has the potential to serve as an effective procedure for palliation of recurrent MPE in terminal cancer patients.

Keywords: malignant pleural effusion, permanent catheter, terminal cancer

\section{Introduction}

Malignant pleural effusion (MPE) is a pathological condition caused by cancer. A disruption to the fluid exchange in the pleura leads to pleural effusion (PE). The formation of interstitial pleural fluid increases due to inflammation caused by pleural carcinosis. ${ }^{1}$ It seems that the parietal pleura is more important in terms of fluid exchange than the visceral pleura, due to the parietal pleura's contiguity to lymphatic openings in the pleural cavity and microvessels. ${ }^{2}$ At advanced stages, nearly all tumors can affect the pleura and thereby cause MPE. ${ }^{3}$ In Europe, the frequency of MPE is estimated at $375,000-400,000$ patients per year. ${ }^{3,4}$ Of these cases, most are caused by lung cancer ( $\sim 40 \%$ ) because of the close anatomical contiguity between lung and pleura. ${ }^{5} \mathrm{MPE}$ has a big impact on the quality of cancer patients' lives as it contributes to both symptoms and hospitalization. Most patients complain of chest pain and dyspnea. ${ }^{3}$ The objective of treating MPE is palliation.

Different procedures to relieve the symptoms of PE exist, all with the same aim to drain fluid and may be to prevent relapse. A treatment option is chemotherapy, but only if the primary tumor is chemosensitive. ${ }^{6,7}$ Another procedure is thoracentesis, where dyspnea caused by $\mathrm{PE}$ is relieved. For this procedure, patients need to be hospitalized, which causes inconvenience and increased expenses. ${ }^{3,8}$ Therefore, a permanent chest drain connected to Department of Surgical

Gastroenterology, Aarhus University Hospital, Noerrebrogade 44, Building IC, 8000 Aarhus C, Denmark

Tel +45 28738496

Email michellemeiermd@gmail.com 
a port that the patients themselves can handle at home has been suggested for patients with frequently recurring PE.

To evaluate the effectiveness of implantation of a permanent PleurX catheter (CareFusion catheter system, San Diego, CA, USA) in the treatment of recurrent MPE in patients with terminal cancer disease, we conducted a retrospective study including data on technical success rate, adverse events (AEs), and catheter patency.

\section{Patients and methods}

We conducted a retrospective study of all consecutive terminal cancer patients who had a permanent PleurX catheter implanted because of recurrent MPE at our institution in the period from February 2014 to August 2015.

The study was approved by the Danish Data Protection Agency (record number 1-16-02-556-14), and the requirement to obtain informed consent from patients was waived due to the retrospective nature of the study and the high morbidity prior to the data collection.

\section{Study population}

Eighteen consecutive patients with recurrent MPE who had a permanent PleurX catheter implanted were identified by searching the patient administrative system. No patients were excluded.

\section{Data on patients and procedures}

Medical records were reviewed, and the following data were acquired: age, sex, primary disease, treatment of primary disease, symptoms related to $\mathrm{PE}$, treatment of $\mathrm{PE}$ prior to permanent catheter implantation, localization of implantation, technical success, AEs, length of hospitalization, readmissions, catheter patency, and residual lifetime after catheter implantation.

The indication for implantation of a permanent PleurX catheter was recurrent MPE. Contraindications for implantation were coagulopathy. Technical success was defined as successful placement of the catheter with drainage of PE. The follow-up period was defined from the date of procedure start to the end of data collection in October 2015, to catheter removal without reimplantation, or to the death of the patient. AEs were rated according to the Common Terminology Criteria for Adverse Events version $4.0^{9}$ and classified as intra-procedural AEs, early AEs (postprocedural days 1-7), and late AEs ( $>7$ days after the procedure).

\section{Technique of catheter implantation}

Two trained interventional radiologists performed the placement of the permanent PleurX catheters. Preprocedural PE was identified by chest X-ray or ultrasonography. The

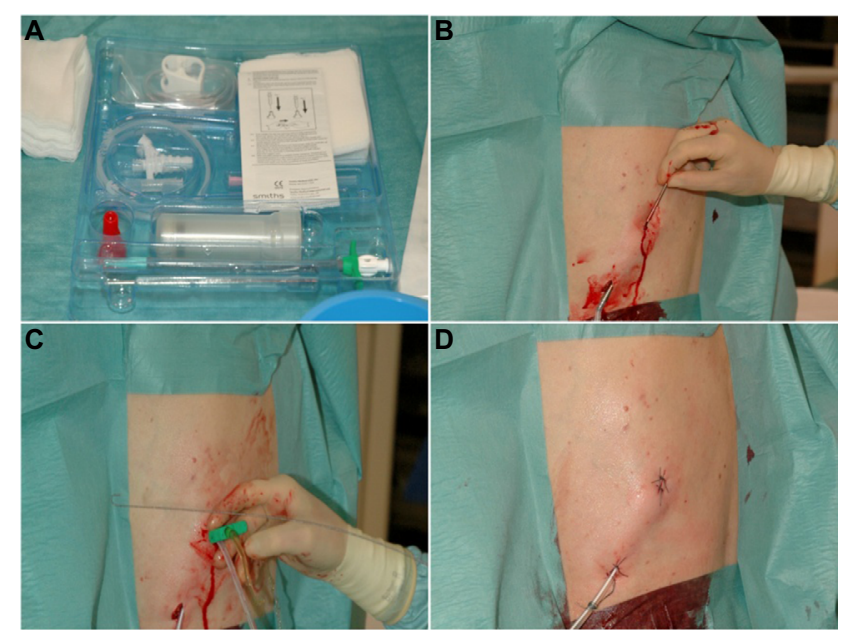

Figure I (A-D) A procedure has earlier been described by the group when implanting a permanent peritoneal PleurX catheter. ${ }^{18}$

Notes: The procedure is here briefly described for the implantation of a pleural PleurX catheter performed in local anesthesia (Xylocaine 1\%) and under sterile conditions using the PleurX catheter kit (A). Two skin incisions are made, one medial for guide wire insertion and one $\sim 5-8 \mathrm{~cm}$ lateral and caudal to the first incision for catheter exit. The fenestrated end of a I5.5-G pleural catheter is tunneled subcutaneously from the caudal lateral to the cranial medial incision crossing the costophrenic sulcus (B). Under US guidance, a J-Tip guide wire is inserted into the pleural space by an I8-G needle through the inferior incision. The needle is removed and a 16-G peel-away introducer is passed over the guide wire, and the guide wire is removed (C). The subcutaneously placed fenestrated end of the catheter is inserted into the peel-away introducer and further into the pleural space, and the peel-away introducer is removed. Both incisions are sutured. The catheter is sutured to the skin (D), connected to the catheter bag, and opened to ensure flow. Bandages are applied.

procedure was performed using the PleurX catheter kit as illustrated in Figure 1. An interventional suite was booked for 45 minutes. One interventional radiologist and two nurses were required for the procedure. After the procedure, verbal instructions, written information, and a DVD with instructions were given to the patient, family, or caretakers.

\section{Trial registration}

Trial registration was not relevant for this study.

\section{Results}

Eighteen consecutive patients (6 males, 12 females) had a permanent PleurX catheter implanted treating terminal malignant cancer disease with recurrent PE (Table 1).

Symptoms of PE included dyspnea at rest $(72 \%)$ or with exertion $(22 \%)$, cough $(22 \%)$, thoracic pain $(17 \%)$, fatigue (17\%), and dizziness (6\%).

Out of the 18 patients, 14 patients $(78 \%)$ had had more than five pleurocenteses performed prior to implantation of the permanent catheter. Two patients (11\%) had talc pleurodesis.

In total, 22 permanent PleurX catheters were implanted (right: $n=12$ [54.5\%], left: $n=10$ [45.5\%]) with a technical success rate of $100 \%$. Two patients had a permanent pleural catheter implanted bilaterally. Two patients had catheters 
reimplanted. Eleven procedures $(50 \%)$ were inpatient procedures with a median length of hospitalization of 1.5 days (range: 1-7 days), while eleven procedures (50\%) were performed in an outpatient setting. Procedure-related AEs and catheter patency are shown in Table 2 .

In total, eight patients $(44,4 \%)$ experienced AEs, out of these patients only three experienced more than one AE (Table 2). Six patients (33.3\%) experienced minor AEs, including three patients with pneumothorax in the early postprocedural period

Table I Descriptive characteristics of 18 consecutive patients who had a permanent PleurX catheter implanted because of recurrent MPE

\begin{tabular}{ll}
\hline Characteristic & \\
\hline Age (median), years (range) & $68(24-95)$ \\
Sex, male:female (ratio) & $6: 12(0.5)$ \\
Primary disease, $\mathrm{n}_{\text {patients }}(\%)$ & \\
C. mammae & $4(22.2)$ \\
Mesothelioma & $2(11 . \mathrm{I})$ \\
Non-small-cell lung cancer & $2(\mathrm{II.I})$ \\
Small cell lung cancer & $\mathrm{I}(5.6)$ \\
C. coli & $\mathrm{I}(5.6)$ \\
Sarcoma & $\mathrm{I}(5.6)$ \\
C. cervix uteri & $\mathrm{I}(5.6)$ \\
Urothelial carcinoma & $\mathrm{I}(5.6)$ \\
Melanoma & $\mathrm{I}(5.6)$ \\
Renal cell carcinoma & $\mathrm{I}(5.6)$ \\
C. anii & $\mathrm{I}(5.6)$ \\
C. ovarii & $\mathrm{I}(5.6)$ \\
Signet ring cell carcinoma of esophagus & $\mathrm{I}(5.6)$ \\
\hline
\end{tabular}

Abbreviations: C, cancer; MPE, malignant pleural effusion.

Table 2 Procedure-related AEs in 18 consecutive patients who had a permanent PleurX catheter implanted because of recurrent MPE

\begin{tabular}{llll}
\hline AEs & Grade $^{\mathbf{a}}$ & $\mathbf{n}_{\text {catheters }}$ & $\%$ \\
\hline $\begin{array}{l}\text { Intra-procedural } \\
\quad \text { None }\end{array}$ & 22 & 0.0 \\
$\begin{array}{l}\text { Early (postprocedural days I-7) } \\
\quad \text { Pneumothorax (<2 cm from the chest }\end{array}$ & $\mathrm{I}$ & 3 & 13.6 \\
$\quad$ wall) & & \\
$\quad$ Soreness at the site of catheter access & $\mathrm{I}$ & 2 & 9.1 \\
Late (more than postprocedural day 7) & & & \\
$\quad$ Soreness at the catheter access site & $\mathrm{I}$ & 2 & 9.1 \\
$\quad$ Catheter dislocation & 3 & $\mathrm{I}$ & 4.5 \\
$\quad$ Asymptomatic loculation & 3 & $\mathrm{I}$ & 4.5 \\
$\quad$ Infection at the catheter access site & 2 & $\mathrm{I}$ & 4.5 \\
$\quad$ Leakage at the catheter access site & $\mathrm{I}$ & $\mathrm{I}$ & 4.5 \\
$\quad$ Clogging of catheter & $\mathrm{I}$ & $\mathrm{I}$ & 4.5 \\
Catheter patency & & & \\
$\quad$ Functional & & 20 & 90.9 \\
$\quad$ Nonfunctional & 2 & 9.1 \\
\hline Nota
\end{tabular}

Notes: a Graded according to the Common Terminology Criteria for Adverse Events version 4.0. ${ }^{9}$ Grade I: minor, Grade 2: moderate, Grade 3: severe, Grade 4: life-threatening, and Grade 5: death.

Abbreviations: $A E$, adverse event; MPE, malignant pleural effusion. not requiring any further interventions. Two of these patients and two other patients experienced transient soreness at the catheter access site. One incident of leakage at the catheter access site $\left(\mathrm{n}_{\text {patient }}=1\right)$ spontaneously stopped and did not require any intervention. Infection at the catheter access site occurred in one case and was treated with antibiotics. Two patients had their dysfunctional catheter removed due to, respectively, asymptomatic loculation and catheter dislocation. Only the latter had a new permanent catheter implanted.

Eight patients (44.4\%) had a postprocedural readmission related to the permanent catheter, including the two abovementioned cases of dysfunctional catheters being removed. Other cause of catheter-related readmissions was a lack of PE into the catheter $\left(\mathrm{n}_{\text {patient }}=1\right)$ due to clogging. Here the catheter was flushed. Two patients experienced dyspnea. The solution to one case was to keep the catheter open more often; in the second case, the catheter was conservatively manipulated in the pleural cavity (no procedural intervention) with the result of drainage of sufficient PE. One patient experienced soreness at the catheter access site well treated with simple analgesics. One patient had accidentally removed the catheter, and a new permanent catheter was implanted. One patient was readmitted because of lack of PE from the catheter, which was found to be due to no further fluid production, and the catheter was removed.

Two patients were alive at the end of the follow-up period, and their catheters were still functioning, being implanted for a median of 129.5 days. Fourteen patients died during the follow-up period with a median residual lifetime after a permanent catheter implantation of 45 days. No deaths occurred in relation to the permanent catheter. Two patients had their catheter discontinued before the end of the follow-up period.

\section{Discussion}

In this study of 18 consecutive patients with terminal cancer disease and recurrent MPE, we demonstrated that implantation of a permanent catheter for MPE could serve as an effective procedure for palliation.

The progress of MPE results in a variety of symptoms where dyspnea by far is the most important. ${ }^{10}$ For most patients, pleural carcinosis results with a very short life expectancy of a few months. ${ }^{3}$ Fourteen of the 18 patients in the present study died during the follow-up period. The median residual lifetime after implantation of the pleural catheter was 45 days. Relief of symptoms in these patients is very important to obtain a certain quality of life $(\mathrm{QoL})$, and ideally, this should be obtained by methods not acquiring hospitalization and without a high frequency of AEs. 
In conclusion, all of these criteria are met by the implantation of a permanent PleurX catheter as demonstrated in the present study.

In the present study, we found no procedural difficulties, and none of the 18 patients had to be drained or treated for MPE by other modalities.

In total, 22 catheters were implanted in 18 individuals with MPE. Of these, $100 \%$ were implanted with initial success. All patients experienced relief of symptoms. Similar high technical success rates have been documented in other studies. ${ }^{10}$ Tremblay and Michaud ${ }^{11}$ demonstrated similar high figures in a single-center experience with 250 tunneled pleural catheter insertions for MPEs.

Eight patients (44.4\%) experienced AEs; three patients experienced more than one AE. In six patients (33.3\%), the AEs were minor, including three patients with pneumothorax not requiring any further treatment and four patients with soreness at the catheter site treatable with simple analgesics. One patient experienced both a moderate and a severe AE. The patient had infection at the catheter side, which was treated with antibiotics, and later developed loculation in the pleural cavity, which made it necessary to remove the catheter. Complication rates in the present study were comparable to those found in other studies dealing with indwelling of pleural catheters ${ }^{10,11}$ and less than seen in chemical and talc pleurodesis. ${ }^{12,13}$ None of the patients in the present study experienced empyema, a feared complication after the placement of pleural catheters. ${ }^{14}$

Implantation of PleurX catheter is a minimal invasive procedure with the potential to be performed in an outpatient setting. In the present study, $50 \%$ of the procedures were performed in the outpatient clinic and 50\% were inpatient procedures with a median length of hospitalization of 1.5 days. These figures are in agreement with a study by Putnam. ${ }^{5}$

We did not perform a cost-benefit analysis of the PleurX catheter for the treatment of MPE. However, in a study by Puri et al, ${ }^{15}$ they concluded that implantation of PleurX catheters for MPE was cost-effective compared to other treatment modalities, especially in patients with a short life expectancy. This was confirmed by Myers and Michaud. ${ }^{16}$

Common malignancies causing MPE include mesothelioma, breast cancer, lung cancer, gastrointestinal cancers, and gynecological cancers. In most instances, MPE is a complication of far-advanced cancer. In this sense, patients in the present study represent a typical cohort of patients with MPE, ie, they had the typical cancers and all far advanced. ${ }^{17}$

\section{Limitations}

Limitations exist when interpreting our results. Because of the retrospective design, caution should be taken concerning possible selection and information bias. Using the Danish National Patient Registry allows, however, complete followup of the 18 patients who were successfully included, which strengthens this study. Other limitations to this study are of course our small population size and the lack of a control group. In the future, we need to conduct prospective studies including a larger number of patients to evaluate the implantation of pleural catheters. Ideally, information regarding QoL in terms of a validated questionnaire would be optimal in the prospective design; however, the follow-up needs to be early after implantation of the catheter due to the very short life expectancy of this group of patients. Efficacy and cost-benefit analysis should be made and compared to other possible modalities for the treatment of MPE.

\section{Conclusion}

Our study shows that implantation of a permanent PleurX catheter is a safe procedure with a high success rate and only few AEs. The procedure has the potential to serve as an effective procedure for palliation of recurrent MPE in terminal cancer patients who have a very short residual life expectancy. This study also indicates that the procedure can be performed in an outpatient setting.

\section{Acknowledgment}

We used the same procedures as in our previous research "Malignant ascites in patients with terminal cancer is effectively treated with permanent peritoneal catheter" published in Acta Radiol Open, 2015.

\section{Disclosure}

The authors report no conflicts of interest in this work.

\section{References}

1. Weick JK, Kiely JM, Harrison EG, Carr DT, Scanlon PW. Pleural effusion in lymphoma. Cancer. 1973;31(4):848-853.

2. Noppen M, De Waele M, Li R, et al. Volume and cellular content of normal pleural fluid in humans examined by pleural lavage. Am J Respir Crit Care Med. 2000;162(3 pt 1):1023-1026.

3. Loddenkemper R. [Management of malignant pleural effusions] Management der malignen Pleuraergüsse. Pneumologie. 2005;59(2):120-135.

4. Mishra E, Davies HE, Lee Y. Malignant pleural disease in primary lung cancer. In: Sprio SG, Huber RM, Janes SM, editors. Thoracic Malignancies. UK: European Respiratory Society Journals Ltd; 2009;313-318.

5. Putnam JB. Malignant pleural effusions. Surg Clin North Am. 2002;82(4):867-883.

6. Hammerschmidt $\mathrm{S}$, Wirtz H. Lung cancer: current diagnosis and treatment. Dtsch Arztebl Int. 2009;106(49): 809-818; quiz819-quiz820. 
7. Fujita A, Takabatake H, Tagaki S, Sekine K. Combination chemotherapy in patients with malignant pleural effusions from non-small cell lung cancer: cisplatin, ifosfamide, and irinotecan with recombinant human granulocyte colony-stimulating factor support. Chest. 2001;119(2): 340-343.

8. Sahn SA. Malignant pleural effusions. Clin Chest Med. 1985;6: $113-125$

9. US National Institutes of Health. Common Terminology Criteria for Adverse Events (CTCAE) Version 4.0. US National Institutes of Health; 2009. Available from: http://evs.nci.nih.gov/ftp1/CTCAE/ CTCAE_4.03_2010-06-14_QuickReference_5x7.pdf. Accessed August $17,2016$.

10. Kaifi JT, Toth JW, Gusani NJ, et al. Multidisciplinary management of malignant pleural effusion. J Surg Oncol. 2012;105(7):731-738.

11. Tremblay A, Michaud G. Single-center experience with 250 tunnelled pleural catheter insertions for malignant pleural effusion. Chest. 2006; 129(2):362-368.
12. Walker-Renard PB, Vaughan LM, Sahn SA. Chemical pleurodesis for malignant pleural effusions. Ann Intern Med. 1994;120(1):56-64.

13. Viallat JR, Rey F, Astoul P, Boutin C. Thoracoscopic talc poudrage pleurodesis for malignant effusions. A review of 360 cases. Chest. 1996; 110(6):1387-1393.

14. Lee SF, Lawrence D, Booth H, Morris-Jones S, Macrae B, Zumla A. Thoracic empyema: current opinions in medical and surgical management. Curr Opin Pulm Med. 2010;16(3):194-200.

15. Puri V, Pyrdeck TL, Crabtree TD, et al. Treatment of malignant pleural effusion: a cost-effectiveness analysis. Ann Thorac Surg. 2012;94(2):374-380.

16. Myers R, Michaud G. Tunneled pleural catheters: an update for 2013. Clin Chest Med. 2013;34(1):73-80.

17. Heffner JE, Klein JS. Recent advances in the diagnosis and management of malignant pleural effusions. Mayo Clin Proc. 2008;83(2):235-250.

18. Meier M, Mortensen FV, Madsen HHT. Malignant ascites in patients with terminal cancer is effectively treated with permanent peritonealcatheter. Acta Radiol Open. 2015;4(7):2058460115579934.
Cancer Management and Research

\section{Publish your work in this journal}

Cancer Management and Research is an international, peer-reviewed open access journal focusing on cancer research and the optimal use of preventative and integrated treatment interventions to achieve improved outcomes, enhanced survival and quality of life for the cancer patient The manuscript management system is completely online and includes

\section{Dovepress}

a very quick and fair peer-review system, which is all easy to use. Visit http://www.dovepress.com/testimonials.php to read real quotes from published authors. 